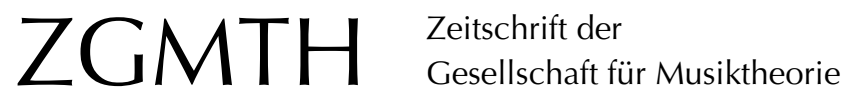

Dreyer, Hubertus / Meidhof, Nathalie (2014): VIII. European Music Analysis Conference (EuroMAC), Leuven, 17.-20. September 2014. ZGMTH 11/2, 267-269. https://doi.org/10.31751/734

C 2014 Hubertus Dreyer, Nathalie Meidhof

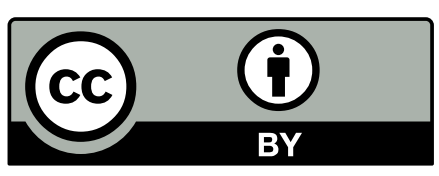

Dieser Text erscheint im Open Access und ist lizenziert unter einer Creative Commons Namensnennung 4.0 International Lizenz.

This is an open access article licensed under a Creative Commons Attribution 4.0 International License.

veröffentlicht / first published: 24/07/2015

zuletzt geändert / last updated: 19/02/2016 


\section{European Music Analysis Conference (EuroMAC), Leuven, 17.-20. September 2014}

»EuroMAC celebrates its 25th anniversary« - so heißt es zu Beginn des Vorworts zum Programmheft der VIII. European Music Analysis Conference in Leuven/Belgien von Pieter Bergé, dem Präsidenten des Organisationskomitees. Der Anlass versteht sich zugleich als Auftrag. Getragen von sieben europäischen Schwestergesellschaften für Musiktheorie und musikalische Analyse, darunter der deutschsprachigen GMTH, soll EuroMAC weiterhin ein Forum bleiben, das die große Themenvielfalt in der Musiktheorie zusammenführt. Angestrebt wird aber auch eine neue Form, die unter Wahrung der Pluralität stärker als bisher den Diskurs und die Weiterentwicklung von Themenschwerpunkten fördert. Konzept und Durchführung des Kongresses waren in vieler Hinsicht beeindruckend und innovativ, beginnend mit der medialen Infrastruktur: Im Vorfeld wurden neue Medien - wie von der Kongressleitung eigens eingerichtete Internetforen - genutzt, während des Verlaufs waren Materialien sowie ein Newsfeed ständig online verfügbar. Auch das pulsierende und vielgestaltige Leben der Hochschulstadt Leuven und der geschichtsträchtige Veranstaltungsort - der innerstädtische Campus der KU Leuven und ihre Musikwissenschaftliche Abteilung -, trugen zusammen mit Kleinigkeiten wie herrlichem Herbstwetter nicht unwesentlich zum Gelingen bei. Gleich die Eröffnung im traditionsreichen Promotion Room und die anschließende Beer Reception in der University Hall gerieten zu einem stimmungsvollen Einstieg in diese Jubiläumskonferenz, die auch im weiteren Verlauf mit bestens organisierten Kaffeepausen und einem geselligen Conference Dinner aufwarten konnte.

Insbesondere aber betraf das veränderte EuroMAC-Konzept den organisatorischen
Rahmen der Vorträge: Zum großen Teil fanden sie in »Pre-organized Sessions" statt - einem Format, das diesen Kongress entscheidend prägte. Die einzelnen Beiträge wurden von Anfang an zu thematisch zusammengehörigen Blöcken gebündelt. Daraus ergaben sich gleichsam kleine Konferenzen innerhalb der Konferenz, jeweils eingeleitet durch einen Vortrag, der das Thema umriss, oft beschlossen durch Gesprächsrunden. Dieses Konzept eröffnete den Raum für fokussierten fachlichen Austausch und zahlreiche interessante Diskussionen. Eine Reihe der Sessions befasste sich mit speziellen, teilweise erst in der Entwicklung befindlichen Analysezugängen. Sessions wie "Analysing Performance and Performing Analysis : Synergies and Interactions between Musical Analysis and Musical Performance", "Agency in Musical Performance», "Music Analysis and the Body", "Corpus Studies, Empirical Methods, and the Analysis of Musical Form«, »Musical Schemata and Historically Informed Listening", „Computational Music Analysis«, »Algebraic Combinatorics of Scales and Modes with Applications to Music Analysis" und "Topic Theory and Music Analysis" stellten nicht nur neue Forschungsbereiche vor, in ihnen zeigte sich auch besonders deutlich eine der wesentlichen Tendenzen, die den Kongress durchzogen: Immer häufiger erweisen sich Themen als fruchtbar, die auf dem Grenzland der Musiktheorie zu Nachbardisziplinen angesiedelt sind.

Andere Sessions widmeten sich bestimmten Korpora (»Analysing Popular Music: a View from 2014 ", »In the Shadow of the Pantheon: Analysing Minor Masters and Secondary Composers", »Analyzing the Renaissance Polyphonic Mass and Office of the Dead", "Words and Music in English Restoration Op- 
era: Analytical Perspectives", "Jazz Harmony: Theory and Practice«). Eindrucksvoll zu erleben waren namentlich die Entwicklungen in der Analyse von Jazz und Popularmusik, teils Frucht eines Bewusstseinswandels, der z.B. das kompositorische Schaffen des Jazzmusikers Wayne Shorter als geeigneten Gegenstand einer Korpusstudie erscheinen lässt, teils - wo es primär um weder notierte noch notierbare Aspekte der Musik geht - durch Fortschritte der Computertechnologie ermöglicht.

Traditionelle musiktheoretische Themen unter einem neuen Blickwinkel wurden in einer ganzen Reihe von Sessions erörtert (»Fétis and Music Theory", „Non-Verbal Theories: Partimento and Craft Learning in the 18th and 19th Centuries«, »Tonality: Recent Theoretical Models and Analytical Tools", »Meter in the Moment", "Analysing Rameau - Rameau Analysing", "Schenker's Formenlehre", »Form, Linear Analysis, and Interpretative Context«). Vielfach wurde auch die Relevanz von analytischen Zugängen für den aktuellen Diskurs beziehungsweise für Werke der Neuen Musik thematisiert (»Form/Structure and Continuity/Discretization: What Principles for Music Analysis Today?", »Analysis Beyond Notation in 20th and 21st Century Music", "Analysing 'Un-Analysable`Art Music Since 1950«, "Listening to Electroacoustic Music through Analysis«, "Harmonic Plasticity and the Modelling of Musical Motion in Tonal, Post-Tonal and Jazz Idioms", »In Search for the Structural Principles of Harmony«). Ein weiteres und sicher noch ausbaufähiges Themenfeld betraf den Einbezug von regionalen Musikkulturen und ihrer Analyse (»No Orientalisms! Four Regional Approaches to Harmony: Russian, Azerbaijani, Polish and Chinese«, »Dissenters beyond the Centres: Analytical Perspectives on Greek Musical Modernism«).

Hinter den sehr vielfältigen Diskursen trat freilich zuweilen die Bedeutung des Einzelvortrages zurück - im Extremfall so weit, dass die Vorträge nur das Sprungbrett für eine ausführliche Diskussion des jeweiligen Themas bildeten (so in "Music Analysis and the Body", einer Session, die mit der Gründung einer lose gefügten Forschungsgruppe beschlos- sen wurde). So erwies sich das Konzept der »Pre-composed Sessions" zwar insgesamt als zukunftsweisend, strukturierend und außerordentlich gewinnbringend, doch mag man sich als Aufgabe für zukünftige Kongresse wünschen, dieses Format, das 'größere (und damit tendenziell auch im angloamerikanischen Raum beheimatete) Diskurse favorisiert, mit den auf eigenständige Qualität gerichteten Ansprüchen und Möglichkeiten freier Einzelvorträge in Einklang zu bringen. Derartige Beiträge erschienen in dieser EuroMAC jedenfalls - zu teilweise sehr kurzen »Post-composed Sessions « zusammengefasst - in der Minderheit. Sie behandelten Themenfelder, die bis auf wenige, durch ihre praktische Relevanz für den Hochschulalltag gewichtige Ausnahmen (wie z. B. »Music Theory Pedagogy« und "Western Music [Theory] in China \& Japan«), etablierten Bereichen zugeordnet wurden (»Form Analysis", "Schoenberg, Webern und die Folgen«, "Harmony 19th-early 20th Century", »Music Theory around 1900«, "Counterpoint«, »Analyses of 20th-Century Compositions", »Theories \& Concepts for the Analysis of 20th-Century Music», „Popular Genres", "Schenkerian Theory", "Music and Film«).

Einige bemerkenswerte Vorträge außerhalb der Sektionen waren die "Guest Lectures». Hier erhielten alle teilnehmenden Gesellschaften Raum für einzelne Beiträge, die - vielleicht angesichts der zeitlichen Terminierung zu Beginn des Kongresstages und der Parallelität von jeweils zwei Vorträgen, vielleicht auch, weil die sehr große Menge der Anregungen die Aufnahmekapazität selbst er interessiertesten Teilnehmer zu überfordern tendierte - freilich nicht immer ein größeres Publikum erreichen konnten. Ariane Jeßulat sprach als Vertreterin der GMTH in einem perspektivenreichen, auch soziologische Aspekte einbeziehenden Vortrag über »Polyphonic Gestures between Architecture and Interaction«. Die neu beteiligte Russische Gesellschaft für Musiktheorie gestaltete eine abendfüllende »Russian Music Theory Welcome Session«.

Ergänzt und umrahmt wurden diese Sessions von zwei "Keynote Lectures" in Form von prominent besetzten Porträts: Hermann 
Danuser eröffnete die Konferenz mit seinem Vortrag »Horizons of Metamusic: The Case of Richard Strauss", in dem er das Potenzial metamusikalischer Fragestellungen erörterte und so ein Plädoyer für eine andere Perspektive auf Strauss' Musik im Speziellen und Analyse im Allgemeinen hielt; Richard Taruskin gab zum Abschluss des Kongresses seine persönliche und nicht von Polemik freie Antwort auf die Frage »Is Anything Unanalysable? « - durchaus polarisierend, doch mit einem versöhnlichen Schlusswort, das auch das wohl größte Verdienst dieses Kongresses gut auf den Punkt brachte: Er, Taruskin, habe hier in Europa, in Leuven, eine Vielfalt musiktheoretischer Ansätze und Diskurse erlebt, wie er sie sich in den USA nicht vorstellen könne. Zu ergänzen wohl: Wie sie überhaupt in keinem einzelnen, in seinen jeweils nationalen Diskursen befangenen Land vorstellbar ist; gerade in der Durchdringung der verschiedenartigen Diskurse aber erweist sich Musiktheorie als ungeheuer facettenreiche, vielfarbige, fruchtbare wissenschaftliche Disziplin.
Die ausgezeichnete Organisation, eine Reihe sehr informativer und neuartiger Beiträge, das zukunftsweisende Format der »Pre-composed Sessions « und nicht zuletzt die interdisziplinären Aspekte ermöglichten einen insgesamt sehr inspirierenden Kongress, der so manche Desiderate, die sich bei vergangenen Kongressen stellten, einzulösen vermochte. Dies alles beizubehalten und zugleich nach Möglichkeit auch verstärkt einige der nationalen Diskurse innerhalb der »European Music Analysis" vorzustellen, die auf diesem Kongress ein gewisses Schattendasein fristeten, zudem noch, ohne die Strukturierung nach »Pre-composed Sessions" zu opfern, auch dem Gewicht querständiger Einzelvorträge wieder größeren Raum zu bieten - das sind sicher nicht leicht zu erfüllende Hoffnungen, die man an eine zukünftige EuroMAC heranbringen mag.

Hubertus Dreyer, Nathalie Meidhof 